\title{
A DYNAMIC ANALYSIS OF MOVING AVERAGE RULES
}

\author{
CARL CHIARELLA*, XUE-ZHONG HE* AND CARS HOMMES**
}

*School of Finance and Economics
University of Technology, Sydney

PO Box 123 Broadway

NSW 2007, Australia

${ }^{* *} \mathrm{CeNDEF}$

Faculty of Economics and Econometrics

University of Amsterdam

Roetersstraat 11, NL-1018 WB

Amsterdam, The Netherlands

Date: Revised Version: April 28, 2005.

An earlier version of this paper was prepared while Tony He was visiting CeNDEF, whose hospitality he gratefully acknowledges. This work has been presented at the CEF-conference, July 8-10, 2004. We would like to thank Hing Hung for his assistance with some of the numerical simulations. The authors would like to thank the referees for their insightful reports and many helpful suggestions. Thanks are also due to the special issue editors Jim Bullard and, in particular, Florian Wagener for providing the proof of the instability part of our main result. The usual caveat applies. Financial supports from the Netherlands Organization of Scientific Research (NWO) under a NWO-MaG Pionier grant, the Australian Research Council (ARC) under a discovery grant, and UTS under a research excellent grant are also gratefully acknowledged.

Corresponding author: Xuezhong (Tony) He, School of Finance and Economics, University of Technology, Sydney, PO Box 123 Broadway, NSW 2007, Australia. Email: Tony.He1@uts.edu.au. Ph: (61 2) 9514 7726. Fax: (61 2) 95147711. 
ABSTRACT. The use of various moving average (MA) rules remains popular with financial market practitioners. These rules have recently become the focus of a number empirical studies, but there have been very few studies of financial market models where some agents employ technical trading rules of the type used in practice. In this paper we propose a dynamic financial market model in which demand for traded assets has both a fundamentalist and a chartist component. The chartist demand is governed by the difference between current price and a (long-run) MA. Both types of traders are boundedly rational in the sense that, based on a fitness measure such as realized capital gains, traders switch from a strategy with low fitness to the one with high fitness. We characterize the stability and bifurcation properties of the underlying deterministic model via the reaction coefficient of the fundamentalists, the extrapolation rate of the chartists and the lag length used for the MA. By increasing the intensity of choice to switching strategies, we then examine various rational routes to randomness for different MA rules. The price dynamics of the moving average rule are also examined and one of our main findings is that an increase of the window length of the MA rule can destabilize an otherwise stable system, leading to more complicated, even chaotic behaviour. The analysis of the corresponding stochastic model is able to explain various market price phenomena, including temporary bubbles, sudden market crashes, price resistance and price switching between different levels.

JEL classifications: D83, D84, E21, E32, C60.

Keywords: Moving Averages, Fundamentalists, Trend Followers, Stability, Bifurcation, Evolutionary Switching. 


\section{INTRODUCTION}

Technical analysts, also known as "chartists", attempt to forecast future prices by the study of patterns of past prices and a few other related summary statistics about security trading. Basically, they believe that shifts in supply and demand can be detected in charts of market movements. In an environment of efficient markets, technical trading rules should not be useful for generating excess returns. However, despite all the evidence presented in academic journals that security prices follow random walks, and consequently that these security markets are at least weak-form efficient, as defined by Fama (1970), the use of technical trading rules still seems to be widespread amongst financial market practitioners.

There have been various studies of the use and profitability of technical analysis. Taylor and Allen (1992) document the enduring popularity of the trading rules in their survey of currency traders in London. Of the respondents, $90 \%$ replied that technical trading rules are an important component of short-term investment strategies. Allen and Taylor (1990) suggest that this is an important finding given the apparent ability of exchange rates to move far from fundamentals over protracted periods of time, as documented by Frankel and Froot $(1986,1990)$. Earlier empirical literature on stock returns finds evidence that daily, weekly and monthly returns are predictable from past returns. Pesaran and Timmermann $(1994,1995)$ present evidence on the predictability of excess returns on common stocks for the S\&P 500 and Dow Jones Industrial portfolios, and examine the robustness of the evidence on the predictability of U.S. stock returns. Brock et al (1992) investigate the sources of the predictability by applying the bootstrap technique to two of the simplest and most popular trading rules, the moving average (MA) and the trading range break rules. They find that returns obtained from buy (sell) signals are not likely to be generated by four popular null models, the random walk, the AR(1), the GARCH$\mathrm{M}$ and the EGARCH models. They document that buy signals generate higher returns than sell signals and the returns following buy signals are less volatile than returns following sell signals. This asymmetric nature of the returns and the volatility of the Dow series over the periods of buy and sell signals suggest the existence of nonlinearities in the data generating mechanism. Recent studies, such as Lo et al (2000), Boswijk et al (2000) and Goldbaum (2003), have also examined explicitly the profitability of technical trading rules and the implications for market efficiency. The profit generating potential of trading rules has also been scrutinised within 
the genetic programming framework by Neely et al (1997) and by the use of artificial neural networks by Gencay (1998) and Fernandez-Rodriguez et al (2000). Griffioen (2003) contains extensive statistical testing of the profitability of technical trading rules, after correcting for transaction costs and data snooping, of many stock market indices including the Dow Jones index.

Most of the cited research has focused on empirical studies. There is also a rapidly expanding related literature on heterogeneous agent models (HAMs) of financial markets, see e.g. the recent surveys by Hommes (2005) and LeBaron (2005) and many references therein. Many of these HAMs have two groups of traders, fundamentalists versus technical analysts. However, most of these models are either complex artificial market simulation models or stylized models in which chartists use oversimplified technical trading rules. This paper develops a simple behavioural HAM with a group of fundamentalists and a group of chartists using a (long-run) MA rule similar to the rules used in financial practice. The technical analysts are assumed to react to buy-sell signals generated by the difference between a long-run and a short-run MA. Both types of traders are boundedly rational in the sense that, based on a fitness measure given by realized capital gains, traders switch from strategies with low fitness to ones with high fitness. The main objectives of this paper are to analyze the stability properties of the model, particularly in relation to the MA trading strategies, and the potential for the model to generate complex dynamics, and to examine the impact of the MA trading rules on the market dynamics.

The plan of the paper is as follows. In the following section, we focus on one of the simplest cases when the fundamentalist demand is determined by mean reversion to the fundamental price, while the technical analyst demand is based on the difference between current price and a MA. Based on certain fitness measures, such as observed differences in payoffs, the traders can make an endogeneous selection of which trading strategies to use, as in Blume et al (1994), Brock and Hommes (1997, 1998), Brock and LeBaron (1996) and Brown and Jennings (1989). Consequently, an adaptive heterogeneous asset pricing model with a market maker scenario is developed. In Section 3, the existence, local stability and bifurcations of the fundamental steady state, in terms of the reaction coefficient of the fundamentalists, the extrapolation rate of the technical analysts, the lag lengths used for the MAs, and switching intensity, are analyzed when the lag lengths of the long MA are small. The analysis, combined with some results on general window length for some special cases, gives us some important insights into the 
effect of increasing the length of the MA. In Section 4 rational routes to randomness, that is, bifurcation routes to complicated asset price dynamics when the switching intensity increases, induced by the MA rule are examined numerically. One of our main findings is that an increase of the window length of the MA rule can destabilize an otherwise stable system, leading to more complicated, even chaotic behaviour. Section 5 introduces a stochastic fundamental price and noise-trader demand processes, and examines the effect of these noise processes when the prices of the corresponding deterministic system are switching between bull and bear markets. This non-linear stochastic model illustrates a range of phenomena observed in real markets such as temporary bubbles, sudden market crashes, price switching between different levels and price resistance. Section 6 concludes the paper.

\section{An Asset Pricing Model with a Market Maker}

Following the framework of Brock and Hommes (1998), this section sets up an asset pricing model with different types of heterogeneous traders who trade according to different trading rules, such as fundamental analysis and technical analysis. The market price is arrived at via a market maker scenario in line with Beja and Goldman (1980), Day and Huang (1990) and Chiarella and He (2003b) rather than the Walrasian scenario used in Brock and Hommes (1998) and Chiarella and He (2002). Whilst the market maker and Walrasian auctioneer mechanisms are highly stylized accounts of how the market price is arrived at, the former may be closer to what is going on in real markets. To focus on the price dynamics of the trading rules, we motivate the excess demand functions of different types of traders by their trading rules directly, rather than deriving the demand functions from utility maximization of their portfolio investment with both risky and risk-free assets (as for example in Brock and Hommes (1998) and Chiarella and He (2002, 2003b)).

Consider an asset pricing model with only one risky asset. Let $P_{t}$ be the price (cum dividend) per share of the risky asset at time $t$. Let $n_{h, t}$ be the market fraction of type $h$ traders at time $t$ with $h=1,2, \cdots, H$ and $\sum_{h=1}^{H} n_{h, t}=1$. Let the excess demand for the risky asset of representative trader from type $h$ at time $t$ be $D_{t}^{h}$. Then the population weighted aggregate excess demand at time $t$ is given by $D_{t}=\sum_{h=1}^{H} n_{h, t} D_{t}^{h}$. We assume that prices are set period by period via a market maker mechanism and adjusted according to the aggregate excess demand 
$D_{t}$, i.e.

$$
P_{t+1}=P_{t}\left[1+\sigma_{\epsilon} \epsilon_{t}\right]+\mu D_{t}=P_{t}\left[1+\sigma_{\epsilon} \epsilon_{t}\right]+\mu \sum_{h=1}^{H} n_{h, t} D_{t}^{h}
$$

where $\epsilon_{t} \sim \mathcal{N}(0,1)$ captures a random excess demand process either driven by unexpected news about fundamentals, or representing noise created by noise traders, $\sigma_{\epsilon} \geq 0$ is a constant and the parameter $\mu>0$ measures the speed of price adjustment (or the aggregate risk tolerance) of the market maker to the excess demand.

For simplicity, we assume throughout this paper that there are only two types of traders: fundamentalists and technical analysts, who in fact are the most widespread types of traders in financial markets and whose trading strategies and excess demand functions are specified in the following discussion. Let the market fraction of fundamentalists and technical analysts at time $t$ be given by, respectively, $n_{f, t}$ and $n_{c, t}$. The population weighted aggregate excess demand $D_{t}$ at time $t$ is then given by $D_{t}=n_{f, t} D_{t}^{f}+n_{c, t} D_{t}^{c}$, where $D_{t}^{f}$ and $D_{t}^{c}$ are the excess demands (to be defined below) of the representative fundamentalist and technical analyst, respectively. Set $m_{t}=n_{f, t}-n_{c, t}$, so that $n_{f, t}=\left(1+m_{t}\right) / 2$ and $n_{c, t}=\left(1-m_{t}\right) / 2$. Using (2.1), the market price of the risky asset is then determined by

$$
P_{t+1}=P_{t}\left[1+\sigma_{\epsilon} \epsilon_{t}\right]+\frac{\mu}{2}\left[\left(1+m_{t}\right) D_{t}^{f}+\left(1-m_{t}\right) D_{t}^{c}\right]
$$

Fundamentalists - The fundamentalists believe that the market price should be given by the fundamental price that they have estimated based on various types of fundamental information, such as earnings, exports, general economic forecasts and so forth. They buy/sell the stock when the current price is below/above the fundamental price. For simplicity, we first assume that ${ }^{1}$ the fundamental price is a positive constant $P^{*}$ and the average excess demand of the fundamentalists is given by $D_{t}^{f}=\alpha\left(P^{*}-P_{t}\right)$, where the parameter $\alpha>0$ is a combined measure of the aggregate risk tolerance of the fundamentalists and their reaction to the mispricing.

Technical Analysts-Unlike the fundamentalists, the technical analysts trade based on charting signals generated from the costless information contained in the history of the price, such

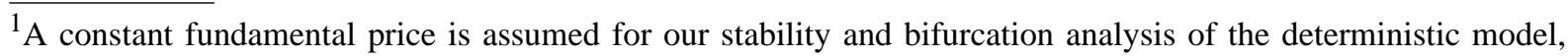
while a random walk fundamental price will be introduced in Section 5 for the stochastic version of the model.
} 
as MAs and various other technical trading rules used in financial markets. The technical analyst average excess demand is here assumed to be based on signals generated by MAs ${ }^{2}$. More precisely, a MA of length $L$ at time $t$ is defined as $m a_{t}^{L}=(1 / L) \sum_{i=0}^{L-1} P_{t-i}$ where $L \geq 1$ is a positive integer. A trading signal is defined as the difference between the current price ${ }^{3}$ and a MA $m a_{t}^{L}$, namely, $\psi_{t}^{L}=P_{t}-m a_{t}^{L}$. For the technical analysts, their average excess demands are assumed to be governed by $D_{t}^{c}=h\left(\psi_{t}^{L}\right)$, where the function $h$ has the general properties $h(0)=0, h^{\prime}(x)>0, x h^{\prime \prime}(x)<0$. This corresponds to one of the very popular technical trading rules whereby technical analysts wish to be long (short) when the current price is above (below) the MA. In this paper, we select $h(x)=\tanh (a x)$ and assume $a=h^{\prime}(0)>0$. Note that this form of technical analyst excess demand function has been used in the literature (e.g. Chiarella(1992)) and it allows us to capture some elements of the filtered MA rules. This is so since, when $a$ is small, the technical analysts initially react cautiously to the long/short signals, in a sense waiting to confirm the maintenance of the change in sign of the signal. In this way they minimize the costs incurred if the signal changes frequently in a short time period. Also, the fact that $-1<h(x)<1$ captures the limited long/short positions, risk averting behaviour and traders' budget constraints.

Fitness Measure and Population Evolution-In order to introduce the adaptive behaviour of agents, we follow the mechanism of Brock and Hommes (1998) and define the fitness functions $\pi_{f, t}, \pi_{c, t}$ as their realized net profit:

$$
\pi_{f, t}=D_{t-1}^{f}\left(P_{t}-P_{t-1}\right)-C_{f}, \quad \pi_{c, t}=D_{t-1}^{c}\left(P_{t}-P_{t-1}\right)-C_{c}
$$

where $C_{f}, C_{c} \geq 0$ are the costs of their strategies. When the number of agents in each group tends to infinity, the population fractions are then updated by the well known logit model probabilities (e.g. Manski and McFadden (1981))

$$
n_{f, t}=\frac{e^{\beta U_{f, t}}}{e^{\beta U_{f, t}}+e^{\beta U_{c, t}}}, \quad n_{c, t}=\frac{e^{\beta U_{c, t}}}{e^{\beta U_{f, t}}+e^{\beta U_{c, t}}},
$$

where

$$
U_{f, t}=\pi_{f, t}+\eta U_{f, t-1}, \quad U_{c, t}=\pi_{c, t}+\eta U_{c, t-1}
$$

\footnotetext{
${ }^{2}$ There is a large practitioner literature on the way MA rules are used to generate buy/sell signals. See for instance Pring (1991) and Neely (1997).

${ }^{3}$ More generally, the current price can be replaced by a short-run MA $m a_{t}^{S}$. For mathematical tractability, we consider the case $S=1$ only in this paper and leave the study of the general case $S>1$ to future work.
} 
and $\eta \in[0,1]$ measures the memory of the cumulated fitness function and $\beta \geq 0$ is the intensity of choice measuring how quickly agents switch between the two strategies. In particular, if $\beta=0$, there is no switching between strategies, while for $\beta=\infty$ all agents immediately switch to the best strategy. See Brock and Hommes (1998) for a more extensive discussion of this switching mechanism.

A Complete Asset Pricing Model_-Based on (2.2) and the above analysis, the market price of the risky asset is determined according to

$$
P_{t+1}=P_{t}\left[1+\sigma_{\epsilon} \epsilon_{t}\right]+\frac{\mu}{2}\left[\left(1+m_{t}\right) \alpha\left(P^{*}-P_{t}\right)+\left(1-m_{t}\right) h\left(P_{t}-m a_{t}^{L}\right)\right]
$$

and, from (2.3)-(2.4), the difference of population fractions $m_{t}$ evolves according to

$$
m_{t}=\tanh \left[\frac{\beta}{2}\left(U_{t}-C\right)\right], \quad C=C_{f}-C_{c} \geq 0
$$

where $\mu \geq 0$ measures the speed of price adjustment of the market maker based on the excess demand, and

$$
U_{t}=\left[D_{t-1}^{f}-D_{t-1}^{c}\right]\left[P_{t}-P_{t-1}\right]+\eta U_{t-1}
$$

with the first term representing the difference in the realized capital gains of the two strategies. Note that we have set $C=C_{f}-C_{c}$ which will be positive if we assume that the fundamentalists incur greater costs than the chartists. By setting $\sigma_{\epsilon}=0$, the nonlinear stochastic dynamical system (2.6)-(2.8) becomes a nonlinear deterministic system where the price follows

$$
P_{t+1}=P_{t}+\frac{\mu}{2}\left[\left(1+m_{t}\right) \alpha\left(P^{*}-P_{t}\right)+\left(1-m_{t}\right) h\left(P_{t}-m a_{t}^{L}\right)\right] .
$$

In general system (2.7)-(2.9) is an $L+2$ dimensional non-linear difference system. We seek principally to understand how its dynamic behaviour is affected by the reaction coefficient $\alpha$ of the fundamentalists, the excess demand function $h$ of the technical analysts, the switching intensity $\beta$, and in particular, the lag length $L$ used for the MA rule.

\section{Stability AND Bifurcation AnAlysis}

In this section, we consider the local stability and local bifurcations of the deterministic system (2.7)-(2.9). The main results are summarized in Proposition 3.1. 
Proposition 3.1. For the deterministic system (2.7)-(2.9), assume $\eta \in[0,1)$. Denote $m^{*}:=$ $\tanh (-\beta C / 2), n_{f}^{*}:=\left(1+m^{*}\right) / 2, n_{c}^{*}:=\left(1-m^{*}\right) / 2$ and $\bar{\alpha}:=\alpha \mu n_{f}^{*}, \bar{a}:=a \mu n_{c}^{*}$.

(i) There exists a unique steady state $\left(P_{t}, m_{t}, U_{t}\right)=\left(P^{*}, m^{*}, 0\right)$, where $P^{*}$ is the constant fundamental price.

(ii) If $\bar{\alpha}=1+\bar{a}$, then the steady state price $P^{*}$ is locally asymptotically stable (LAS) for $0<\bar{a}<L$. At $\bar{a}=L$, there occurs a $1: L+1$ resonance Hopf bifurcation ${ }^{4}$.

(iii) A necessary condition for the steady state price to be LAS is given by $0<\bar{a}<L$ and $0<\bar{\alpha}<2+\bar{a}$ for even $L$ and $0<\bar{\alpha}<2+\frac{L-1}{L} \bar{a}$ for odd L (see Fig. 3.1).

(iv) For all L, $P^{*}$ is LAS if $(\bar{\alpha}, \bar{a}) \in D_{S}(\bar{\alpha}, \bar{a}):=\{(\bar{\alpha}, \bar{a}) ; 2 \bar{a}<\bar{\alpha}<2\}$ (see Fig. 3.1).

(v) For sufficiently large L, $P^{*}$ is unstable if $\bar{a}>\bar{\alpha}$ (see Fig. 3.1 $)^{5}$.

(vi) For $L=1, P^{*}$ is LAS for $(\bar{\alpha}, \bar{a}) \in D_{11}(\bar{\alpha}, \bar{a}):=\{(\bar{\alpha}, \bar{a}) ; 0<\bar{\alpha}<2,0<\bar{a}\}$. In addition, flip and saddle-node bifurcations occur when $\bar{\alpha}=2$ and $\bar{\alpha}=0$, respectively (see Fig. 3.2a).

(vii) For $L=2, P^{*}$ is $L A S$ for $(\bar{\alpha}, \bar{a}) \in D_{12}(\bar{\alpha}, \bar{a}):=\{(\bar{\alpha}, \bar{a}) ; 0<\bar{\alpha}<\bar{a}+2,0<\bar{a}<2\}$. Furthermore, a saddle-node bifurcation occurs when $\bar{\alpha}=0$, a Hopf bifurcation occurs when $\bar{a}=2$, and a flip bifurcation occurs when $\bar{\alpha}=\bar{a}+2$ (see Fig. 3.2b).

(viii) For $L=3, P^{*}$ is $L A S$ for $(\bar{\alpha}, \bar{a}) \in D_{13}(\bar{\alpha}, \bar{a}):=\left\{(\bar{\alpha}, \bar{a}) ; 0<\bar{\alpha}<\frac{2}{3} \bar{a}+2, \bar{a}(2-\bar{\alpha}+\bar{a})<\right.$ 3\}. Furthermore, a saddle-node bifurcation occurs when $\bar{\alpha}=0$, a Hopf bifurcation occurs when $\bar{a}(2-\bar{\alpha}+\bar{a})=3$, and a flip bifurcation occurs when $\bar{\alpha}=\frac{2}{3} \bar{a}+2$ (see Fig. $3.2 c)$.

(ix) For $L=4, P^{*}$ is LAS for $(\bar{\alpha}, \bar{a}) \in D_{14}(\bar{\alpha}, \bar{a}):=\left\{(\bar{\alpha}, \bar{a}) ; 0<\bar{\alpha}<\frac{3}{4} \bar{a}+2,0<\bar{a}<\right.$ $\left.4,(5 \bar{a}-4 \bar{\alpha})(4+\bar{a})^{2}<\bar{a}(8+3 \bar{a}-4 \bar{\alpha})^{2}\right\}$ (see Fig. 3.2d).

\footnotetext{
${ }^{4}$ Resonance bifurcations occur when the complex eigenvalues lie on the unit circle. When $\bar{a}=L$, the eigenvalues are given by $\lambda_{k}=e^{2 k \nu \pi i}$ with $k=1,2, \cdots, L$ and $\nu=1 /(L+1)$. Geometrically, the $L$ eigenvalues correspond to the $L+1$ unit roots distributed evenly on the unit circle, excluding $\lambda=1$. When $L=1$, a flip or perioddoubling bifurcation occurs. When $L=2$, the bifurcation is known as a 1:3 strong resonance, which may lead to two sets of period three cycles with one set stable and other set unstable (e.g. Chiarella and He (2000)). For $L \geq 2$, the bifurcation is accompanied by $1: L+1$ periodic resonances (e.g. Sonis (2000)). For $L_{1}=L_{2}=$ $L=3,4$, instability of the steady state leads to $1: 4$ and 1:5 periodic resonance bifurcations, respectively, and similar dynamics to the 1:3 resonance bifurcation are also found. Theoretical analysis of such types of bifurcation of higher dimensional discrete systems can be exceedingly complicated and is not yet completely understood, (e.g. Example 15.34 in Hale and Kocak (pp. 481-482, (1991))). See Kuznetsov (2004) for an extensive mathematical treatment of bifurcation theory.

${ }^{5}$ We would like to thank Florian Wagener for providing a proof of this result.
} 
A proof of Proposition 3.1 is given in the appendix. Here we discuss some underlying economic intuition using Figures 3.1 and 3.2 illustrating the (in)stability regions and the bifurcation curves. Result (i) of Proposition 3.1 assures that the fundamental price is the unique steady state price and the population fractions of the fundamentalists and chartists at the steady-state are given by $n_{f}^{*}$ and $n_{c}^{*}$, respectively. Obviously, $n_{f}^{*}=n_{c}^{*}=0.5$ when $C=0$. However, if $C>0$, that is costs for fundamentalists' strategies exceed the costs for technical trading rules, then there are more chartists than fundamentalists at the steady state, i.e., $n_{c}^{*} \geq n_{f}^{*}$.

Both parameters $\bar{\alpha}=\alpha \mu n_{f}^{*}$ and $\bar{a}=a \mu n_{c}^{*}$ play an important role in determining the stability/instability of the fundamental price. The market maker's price adjustment speed $\mu$ shows up as a scaling factor in $(\bar{\alpha}, \bar{a})$. Given this scaling factor, $\bar{\alpha}$ and $\bar{a}$ are determined by the population weighted (at the steady state) reaction coefficients of the fundamentalists and chartists, respectively. Intuitively we would expect the fundamentalists to represent a stabilizing force and the activities of the chartists to destabilize an otherwise stable market price. The results of Proposition 3.1 describe how the (local) stability of the market depends on the balance of these forces (captured by $\bar{\alpha}$ and $\bar{a}$ ) and the lag length of the MA, as we explain in the following discussion.

Result (ii) of Proposition 3.1 relates to the stability of the fundamental price along the line $\bar{\alpha}=1+\bar{a}$, for general $L$, as illustrated in Fig. 3.1. This line plays an important role in the stability analysis of the model. Along this line, the stability region is proportionally enlarged as the lag length of the MA process $(L)$ increases. For fixed lag $L$, the stability line segment $\bar{\alpha}=1+\bar{a}$ for $0<\bar{a}<L$ is part of the stability region in the $(\bar{\alpha}, \bar{a})$ parameter plane. To see the economic intuition behind this result, let us examine the price behaviour near the fundamental price. The linearization of (2.6) reduces to $P_{t+1}=P_{t}+\bar{\alpha}\left(P^{*}-P_{t}\right)+\bar{a}\left(P_{t}-m a_{t}\right)$, or equivalently, in deviations $X_{t}:=P_{t}-P^{*}$ from the fundamental price

$$
X_{t+1}=[1+\bar{a}-\bar{\alpha}] X_{t}-\frac{\bar{a}}{L} \sum_{i=0}^{L-1} X_{t-i}
$$

Along the line $1+\bar{a}=\bar{\alpha}$, the stabilizing force from the fundamentalists $(\bar{\alpha})$ and the destabilizing force from the chartists $(1+\bar{a})$ just balance each other. Accordingly, along this line, equation (3.1) becomes $X_{t+1}=-(\bar{a} / L) \sum_{i=0}^{L-1} X_{t-i}$ and stability of the fundamental price is determined exclusively by the MA process. In this case, the stability region of parameter $\bar{a}$ is enlarged as 
the lag for the MA increases. More precisely, local stability is achieved when $\bar{a} / L<1$ (see Chiarella and He (2000) for the mathematical proof of this result).

Based on the above analysis, we may conjecture that the stability region is enlarged as the lag length $L$ increases. However this conjuncture is not true in general and this becomes clear from the results for $L=1,2,3$ and 4. Certainly, a longer MA does reduce the impact of a single period event on chartists' beliefs (and so stabilizes the price process), however the contained price information becomes less significant as the lag length increases. Hence, when both the stable and unstable forces are balanced, as we have just discussed, the stability of the market price is maintained. However, when such forces become unbalanced, particularly with large $\bar{a}$, sudden shifts in demand can trigger an unstable fundamental price, leading to price overshooting, as the lag length increases. This observation is basically the underlying mechanism involved in the change of the local stability region as the lag length of the MA increases. $^{6}$

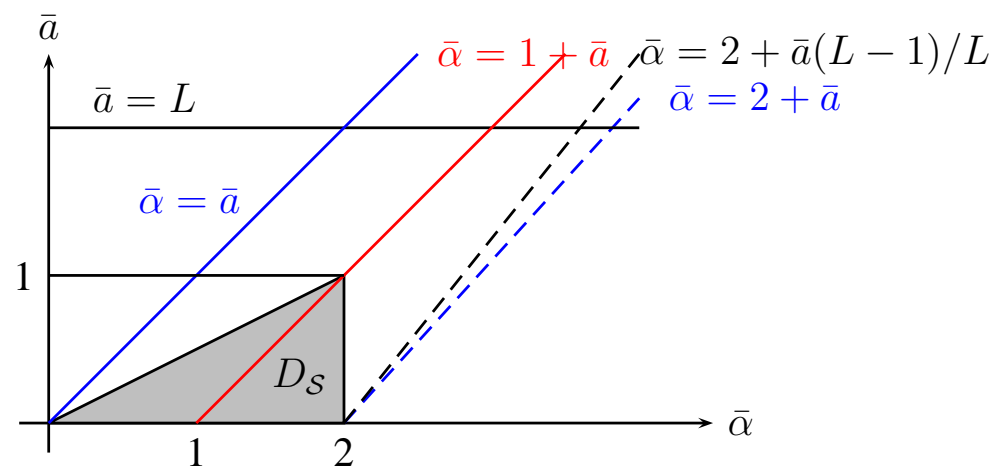

FIGURE 3.1. The common stability region $D_{\mathcal{S}}$ for general lag length $L$ and necessary stability boundaries $\bar{a}=L, \bar{\alpha}=2+\bar{a}$ for even lag $L$ and $\bar{\alpha}=$ $2+\bar{a}(L-1) / L$ for odd lag $L$. On the horizontal (vertical) axis we have the population weighted reaction coefficient of the fundamentalists (chartists) at the steady state, i.e. $\bar{\alpha}:=\alpha \mu n_{f}^{*}, \bar{a}:=a \mu n_{c}^{*}$.

Given the mathematical difficulty in determining the local stability conditions for general lag length $L$ in the $\bar{\alpha}$ and $\bar{a}$ parameter space, it is useful to have some information about the potential unstable regions and common stable regions for all lags. Result (iii) in Proposition 3.1 give us necessary conditions for stability. In other word, the fundamental price is unstable outside the regions that are bounded on the right by the two dotted lines and above by $\bar{a}=L$ in Fig. 3.1. Result (iv) give us sufficient conditions for the stability in terms of $\bar{a}$ and $\bar{\alpha}$ for general

${ }^{6}$ The authors would like to thank an anonymous referee to bringing this point to our attention. 
lag length $L$, and the common stability region $D_{\mathcal{S}}$ is illustrated in Fig. 3.1. It indicates that, for all lag length $L$, the fundamental price is stable when the population weighted coefficients of fundamentalists and chartists are balanced and bounded (i.e. $2 \bar{a}<\bar{\alpha}<2$ ). On the other hand, when the coefficient of the chartists exceeds that of the fundamentalists, result (v) shows that increasing the lag length $L$ in the end destabilizes the system and this is a more interesting result. The intuition for this instability result is the following. Chartist demand depends on the difference between the long-run MA and the current price. As $L$ increases, the MA becomes smoother and more sluggish. When $\bar{a}>\bar{\alpha}$, the relative effect of chartists at the steady state is bigger than that of fundamentalists, a small change in the price leads to a relatively large increase of chartists demand destabilizing the price.

For $L=1,2,3,4$, Proposition 3.1 describes explicitly the regions of LAS in the $(\bar{\alpha}, \bar{a})$ plane and the bifurcation behaviour at the boundaries of those regions where local asymptotic stability turns to instability. These regions are illustrated in Fig. 3.2.

For $L=1$, the technical analysts have no impact on the market price. We go back to the set up of the model and let $m_{t}=1$. Consequently, the price equation is simplified to $P_{t+1}-P^{*}=$ $[1-\alpha \mu]\left(P_{t}-P^{*}\right)$. Hence the stability condition is given by $0<\bar{\alpha}<2$, where $\bar{\alpha}=\mu \alpha$ is the product of the speed of the price adjustment of the fundamentalists towards the fundamental price $(\alpha)$ and the speed of price adjustment of the market maker $(\mu)$. Thus the stability of the steady state price $P^{*}$ is maintained only when the under(over)-reaction from the fundamentalists is balanced by the over (under)-reaction from the market maker. The over-reaction from both may lead to price overshooting, through a flip bifurcation when $\bar{\alpha}=2$.

For $L=2$, the stability region $D_{12}$ and bifurcation boundaries are plotted in Fig. 3.2(b) in the $(\bar{\alpha}, \bar{a})$ parameter plane. The Hopf bifurcation boundary is defined by $\bar{a}=2$ and $\bar{\alpha} \in(0,4)$. For $L=3$, the stability region $D_{13}$ and the bifurcation boundaries are plotted in Fig. 3.2(c). Different from the previous two cases, the Hopf bifurcation now depends on both parameters $\bar{\alpha}$ and $\bar{a}$. For $L=4$, the stability region $D_{14}$ is plotted in Fig. 3.2(d).

For comparison all stability regions $D_{1 L}$ for $L=1,2,3$ and 4 are plotted in Fig. 3.2(e). The changes of the local stability regions as $L$ increases are in line with our previous discussion concerning the stability near the line $1+\bar{a}=\bar{\alpha}$. As $L$ increases, sudden shifts in demand can trigger an unstable price when the reaction speeds are unbalanced. 

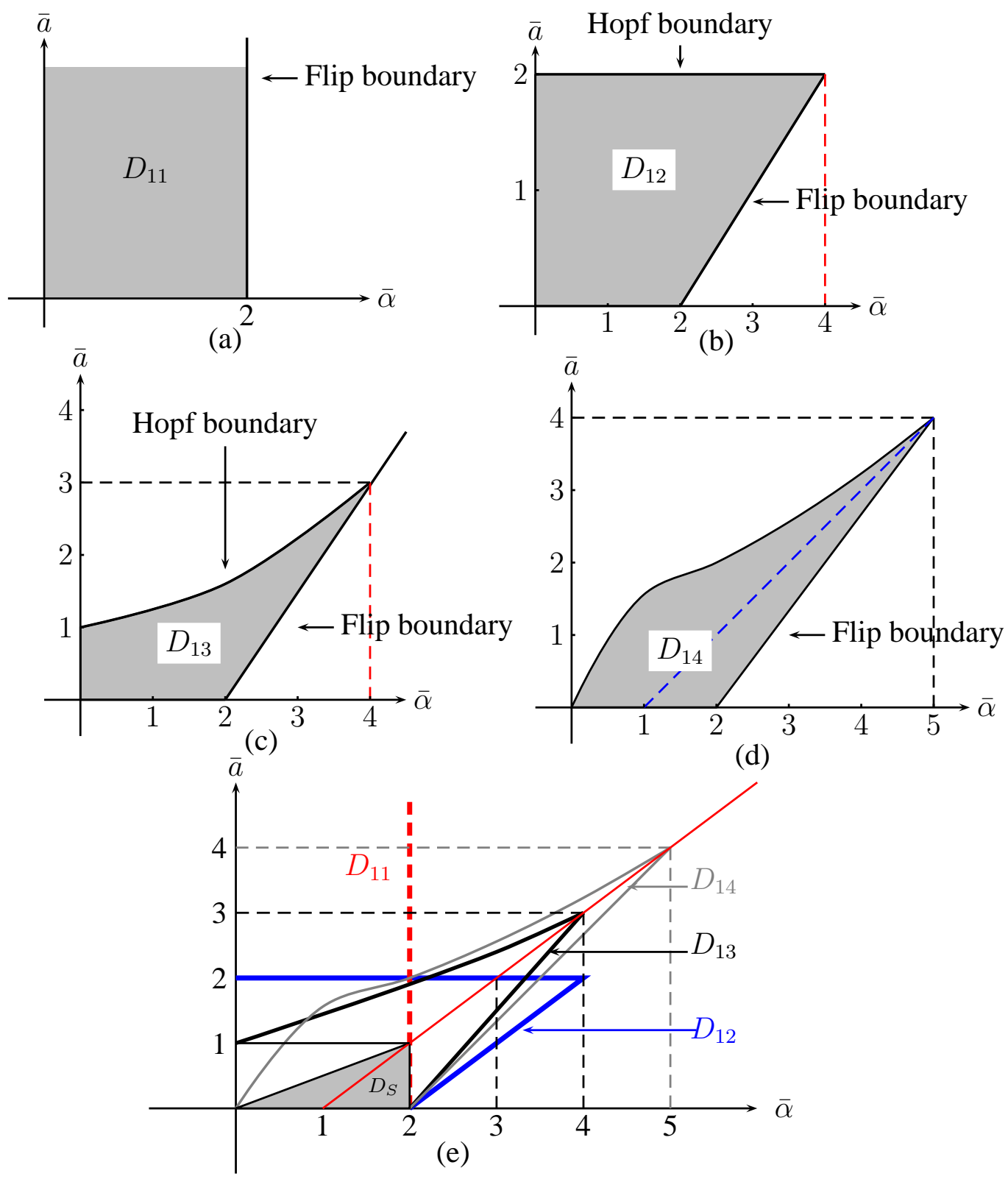

(d)

FIGURE 3.2. Stability regions and bifurcation boundaries for (a) $L=1$, (b) $L=$ 2, (c) $L=3$, (d) $L=4$ and (e) comparison of stability regions and bifurcation boundaries $D_{1 L}$ for $L=1,2,3,4$. On the horizontal (vertical) axis we have the population weighted reaction coefficient of the fundamentalists (chartists) at the steady state, i.e. $\bar{\alpha}:=\alpha \mu n_{f}^{*}, \bar{a}:=a \mu n_{c}^{*}$.

Given the large variety of MA rules used in financial markets and the difficulty of eigenvalue analysis for high-order characteristic equations, it is not clear how different MA rules influence the stability of the steady state price and types of bifurcation that may occur. However the analysis has given some important insights into the fact that local asymptotic stability depends on some subtle balance between the reaction coefficients of fundamentalists and technical analysts. Based on our analysis, we conjecture that as the lag length $L$ increases, the stability region 
tends to shrink towards, but stretch along, the line $\bar{\alpha}=1+\bar{a}$ with common stability region $D_{S}$. This conjecture is partly verified by the numerical simulations in the following section.

Our stability analysis also yields insight as to how the other parameters, the price adjustment factor $\mu$, the intensity of choice $\beta$, the cost difference $C$ between fundamental and technical trading strategies and the lag length $L$ affect the local stability of the fundamental steady state. Increasing the price adjustment factor $\mu$ moves the point $(\bar{\alpha}, \bar{a})$ in Figs. 3.1 and 3.2 in the North-East direction leading either to a flip bifurcation (when the population weighted reaction coefficients of the fundamentalists is relatively large) or to a Hopf-bifurcation (when the population weighted reaction coefficient of the chartists is relatively large). When $C>0$, an increase in $\beta$ leads to an increase in $n_{c}^{*}$, the fraction using the cheap technical trading strategy. Hence, for $C>0$, an increase in $\beta$ moves $\bar{a}$ upwards and $\bar{\alpha}$ downwards, so that the point $(\bar{\alpha}, \bar{a})$ in Figs. 3.1 and 3.2 moves in the North-West direction and the fundamental steady state may lose stability through a Hopf bifurcation. When there is no cost difference between fundamental and chartist strategies, an increase in $\beta$ does not change $\bar{a}$ and $\bar{\alpha}$, so there is no change in the local stability of the steady state. Furthermore increasing $C$ is similar to increasing $\beta$. Finally, the fact that the stability regions become more narrow for higher lags $L$ suggests that an increase in $L$ may destabilize the system, especially when $\bar{a}>\bar{\alpha}$, i.e. the relative impact of chartists at the steady state is larger than that of the fundamentalists. The global dynamics, for different values of the intensity of choice and the lag length $L$, will be investigated in section 4 .

\section{Dynamics of The Nonlinear System}

In this section, we examine the global dynamics of the nonlinear system (2.7)-(2.9) by focusing on the effects of the switching intensity (Subsection 4.1) and of the lag length of the MA (Subsection 4.2).

\subsection{The Effect of The Switching Intensity-Rational Routes to Randomness. Brock and} Hommes (1997, 1998) have proposed simple Adaptive Belief System to model economic and financial markets, where agents adapt their beliefs over time by choosing from different predictors or expectations functions, based upon their past performance as measured by realized profits. Brock and Hommes (1998) show that, as the intensity of choice to switch to better strategies increases, the model is able to generate the entire "zoo" of complex behaviour from local stability to high order cycles and even chaos and this is the so-called Rational Routes to 
Randomness (RRR for short). In this section, we consider the effect of the switching intensity on the price dynamics of the deterministic system (2.7)-(2.9) with two different MAs using $L=4$ and $L=100$. We choose the parameter set $\alpha=1, \mu=2, \eta=0.2, a=1, C=1$.

Note that for $\beta=0$, we have $\bar{\alpha}=\alpha \mu n_{f}^{*}=1$ and $\bar{a}=a \mu n_{c}^{*}=1$, so that for $L=4$ and $\beta=0$, according to Proposition 3.1 (ix) the fundamental price $P^{*}$ is locally stable. On the other hand, since $C>0$, for $\beta=\infty$, we have $\bar{\alpha}=\alpha \mu n_{f}^{*}=0$ and $\bar{a}=a \mu n_{c}^{*}=2$, so that for $L=4$ and $\beta=\infty$, according to Proposition 3.1 (ix) the fundamental steady state is unstable. As the switching intensity $\beta$ increases we therefore expect that the fundamental steady state becomes unstable by a Hopf bifurcation. This is indeed confirmed by numerical simulations as illustrated by the phase plots $\left(P_{t}, m_{t}\right)$, for different values of $\beta=0.2,0.3,0.49,0.52,0.555$ and 0.57 in Fig. 4.1. It is found that, once the fundamental price $P^{*}$ becomes unstable, the solutions converge to figure-eight shaped attractors for low switching intensity (e.g. the cases of $\beta=0.2$ and 0.3 ). Recall that for $L=4$ we have a 6-dimensional system, and the figureeight shaped attractors are in fact 2-dimensional projections of an invariant circle around the unstable fundamental steady state in the 6-dimensional phase space. As the switching intensity increases, the figure-eight shaped attractor grows initially (for $\beta=0.3,0.35$ ) and then stretches to a scissors-shaped attractor (for $\beta=0.49$ ). As the intensity increases further, the simple attractor becomes more complicated (for $\beta=0.52$ ) and eventually leads to strange attractors (for $\beta=0.555$ and 0.57 ). One can see that the market price variation increases as the switching intensity increases. It is interesting to note that these patterns are similar to the rational routes to randomness studied extensively in Brock and Hommes (1997, 1998).

\section{Insert Figure 4.1 Here}

Figure 4.1. Phase plots of $\left(m_{t}, P_{t}\right)$ for $L=4$ and various $\beta=$ $0.2,0.3,0.49,0.52,0.555$ and 0.57 .

For $L=100, \beta=0$, we have $\bar{\alpha}=\alpha \mu n_{f}^{*}=1$ and $\bar{a}=a \mu n_{c}^{*}=1$ and we conjectured earlier that this point lies outside the stability region for $L$ large and this is confirmed by numerical simulations for $L=100$. To illustrate the effect of the switching intensity $\beta$, we include phase plots, in terms of $\left(P_{t}, m_{t}\right)$, for different values of $\beta=0.05,0.1,0.2,0.3,0.35,0.42,0.45$ and 
0.4652 in Fig. 4.2. As $\beta$ increases, the (projection of the) attractor starts with narrow figureeight shapes (for $\beta=0.05$ and 0.1 ) and is then stretched (or extrapolated) by the technical analysts towards the extreme high/low price levels (for $\beta=0.2$ ). The attractors are then broken down to Lorenz-like attractors, similar to those of the celebrated 3-dimensional continuous Lorenz system, see Peitgen et al (1992)) for $\beta$ between 0.3 and 0.35 . As the switching intensity increases further, the Lorenz-like attractors merge into one connected strange attractor (for $\beta=$ 0.42 ) and then to strange attractors (for $\beta=0.45$ and 0.4652 ). Also, as the switching intensity increases, the volatility of both price and population increases.

\section{Insert Figure 4.2 here}

Figure 4.2. Phase plots of $\left(m_{t}, P_{t}\right)$ for $L=100$ and various $\beta=$ $0.05,0.1,0.2,0.3,0.35,0.42,0.45$ and 0.4652 .
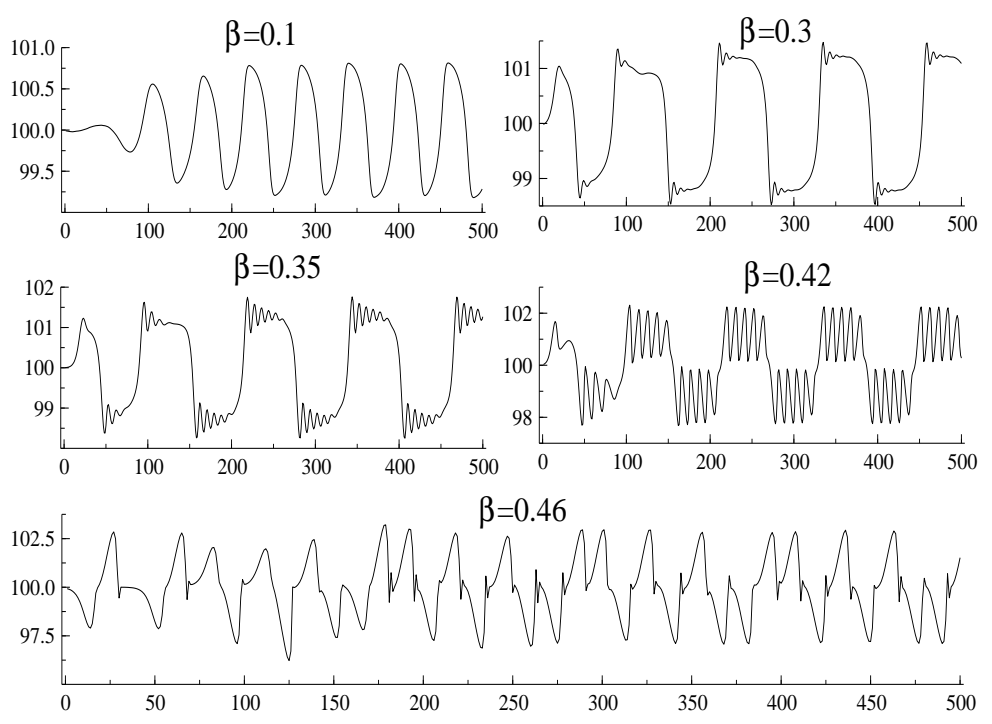

Figure 4.3. Price time series for $L=100$ and $\beta=0.1$ (a), 0.3 (b), 0.35 (c), $0.42(\mathrm{~d})$ and $0.46(\mathrm{e})$.

The corresponding price time series are illustrated for $\beta=0.1,0.3,0.35,0.42$ and 0.46 in Fig. 4.3. One can see that an increase of the switching intensity can generate very interesting price patterns. With a lower switching intensity $(\beta=0.1)$, the fundamental price is unstable and extrapolation of the price trend by the technical analysts pushes the price away from the fundamental price. Because of their limited long/short position, their fitness or utility becomes 
smaller when they reach their limit position. This leads traders to switch back to the fundamental strategy, bringing price back towards the fundamental price. Because of the increase of the fitness of the technical analysts, the price is pushed further beyond the fundamental price to the opposite extreme. As the switching intensity increases (for $\beta=0.3,0.35$ ), such switching from high/low extreme to low/high extreme happens very quickly. At the same time, the price becomes more volatile. This result can be used to explain regular boom and bear markets. As the intensity increases further, the regular switching pattern of the price between two extreme levels is destroyed, leading to highly volatile price patterns (for $\beta=0.46$ ). This phenomenon of the price switching between upper and lower levels gives some economic basis to the notion of upper and lower resistance levels that are frequently discussed in the practitioner literature on technical analysis (see e.g. Pring (1991)).

\subsection{The Effect of the Lag Length-Dynamics of The Moving Average. We now consider}

the effect of the lag length $L$ of the MA rule on the price dynamics of the deterministic system (2.7)-(2.9). As an illustrative example, we choose the parameters $\alpha=1, \mu=2, \beta=0.4, \eta=$ $0.2, a=1, C=0$, for which $\bar{\alpha}=1$ and $\bar{a}=1$. The fundamental price is locally stable for $L=2,3,4$, but it is unstable for $L \geq 5$. Fig. 4.4 illustrates how the phase plot (in terms of $\left.\left(P_{t}, m_{t}\right)\right)$ changes as the lag length $L$ increases.

\section{Insert Figure 4.4 here}

FIgURE 4.4. Phase plots of $\left(m_{t}, P_{t}\right)$ for fixed $\beta=0.4$ and various $L=$ $5,8,9,10,50,90$ and 100 .

For $L=5$, the (2-D projection of the) attractor is given by a figure-eight shaped closed curve with small price variation (about $1 \%$ of the fundamental price level) and there is a tendency among traders to switch from fundamental analysis to technical analysis. For $L=8$, the size of the attractor is enlarged, implying that the deviations of both price and population from the fundamental value, which is $P^{*}=100$ and $n_{c}^{*}=n_{f}^{*}=0.5$, are enlarged. Hence an increase in the MA window $L$ destabilizes the price dynamics. This destabilizing effect becomes more significant when $L$ is increased further to $L=9,10,50$ and the price dynamics become even more complicated for $L=90$ and 100, as indicated by the phase plots in Fig. 4.4.

In order to get more insight into these destabilizing effects of the long-run MA, let us examine the time series of prices and corresponding MAs in Fig. 4.5. It is found that, following a cross 

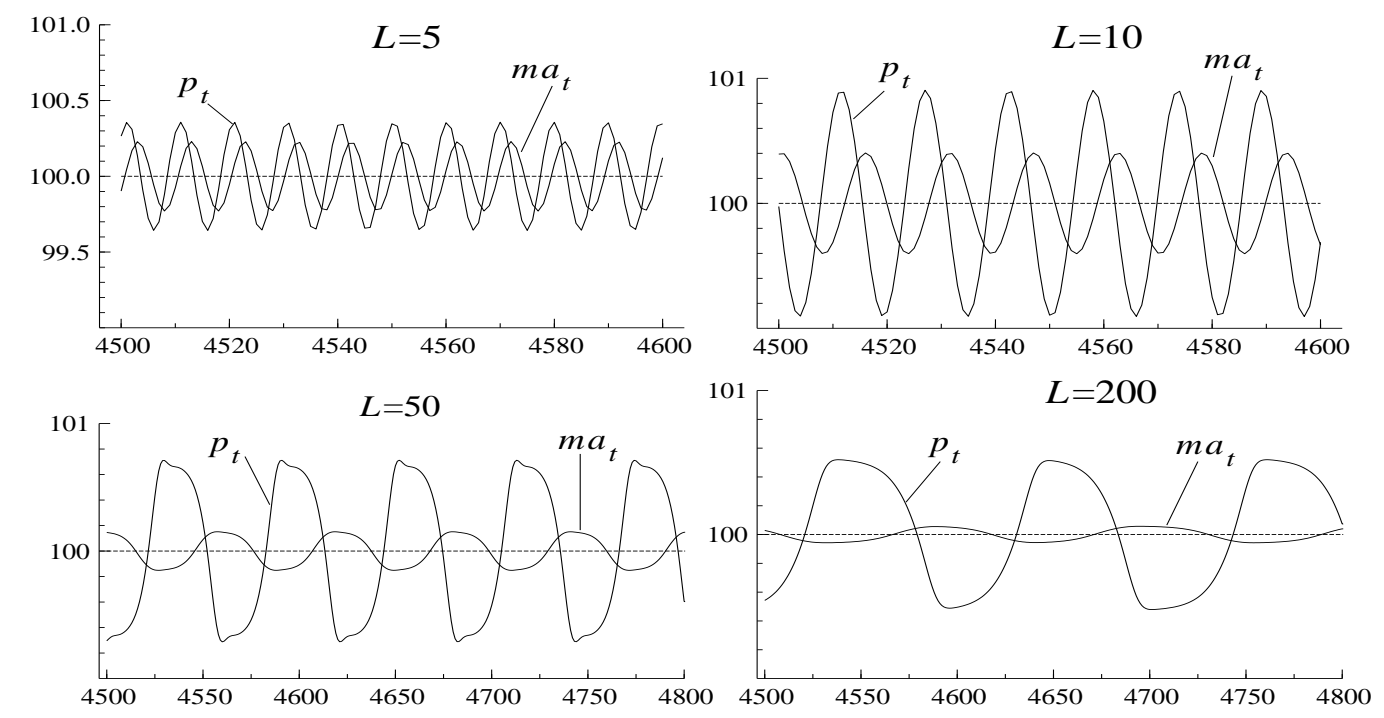

FIGURE 4.5. Price time series for fixed $\beta=0.4$ and various $L=5,10,50$ and 200 .

over of the long run MA and the market price, both the technical analysts and fundamentalists take the same long/short position initially, but soon after they take opposite positions. This helps to accentuate either the up or the down trend, pushing the price to either a higher or a lower level initially, but soon after, their different positions slow down the trend built up initially and bring the price back towards its fundamental level. The time taken for the price to return back to its fundamental value is proportional to the lag $L$. When the lag $L$ for the MA is small, the reversion back to the fundamental happens quickly; as $L$ increases, this reversion takes a longer time.

The destabilizing effect of the lag length $L$ holds in general for the parameters located within regions in which the fundamental price is locally stable for lower lags and unstable for higher lags, as discussed in the above. However, this may not always be the case. As a matter of fact, when the reaction coefficients from both types of traders are carefully balanced (such that $\bar{\alpha}=1+\bar{a})$, an increase of the lag length can stabilize an otherwise unstable system, as indicated in Proposition $3.1^{7}$.

\footnotetext{
${ }^{7}$ Numerical simulations (not reported here) indicate that, in this case, an increase in $L$ can cause an explosive system to become a (locally) stable system.
} 


\section{Time Series Analysis of The Stochastic Model}

The nonlinear dynamic model considered in the previous sections can be treated as the deterministic skeleton of the corresponding stochastic model. The prices observed in real markets are presumably the outcome of the interaction of both non-linear and stochastic elements. Rigorous analytical tools for the analysis of non-linear stochastic dynamical system are still in a development phase (see e.g. Arnold ((1998)) for an up-to-date account). The analytical results that exist deal mainly with affine systems so it seems difficult at the moment to apply these tools to our nonlinear model. In this section we attempt to gain some insights into the behaviour of the nonlinear stochastic model through numerical simulations.

Recall from Section 2, Eq. (2.1) that we already introduced a noise term $\epsilon_{t}$ representing noise created by noise traders. In addition to noisy demand, we also introduce a random walk fundamental price process. We assume that the fundamental price follows a random walk

$$
P_{t+1}^{*}=P_{t}^{*}\left[1+\sigma_{\delta} \delta_{t}\right]
$$

where $\sigma_{\delta} \geq 0$ is a constant measuring the volatility of the return and $\delta_{t} \sim \mathcal{N}(0,1)$. Notice that this specification ensures that relative price changes are stationary.

To illustrate a typical example, we select the parameters $\alpha=0.5, \beta=0.3, a=1, \mu=1, \eta=$ $0.2, C=1, L=100, P_{0}^{*}=P_{0}=\$ 100$. To see the effect of the two noise processes on the price dynamics of the deterministic model, we compare four different cases in terms of $\left(\sigma_{\epsilon}, \sigma_{\delta}\right)$ : (a) $(0,0),\left(\right.$ b) $\left(\sigma_{\epsilon}, 0\right),\left(\right.$ c) $\left(0, \sigma_{\delta}\right)$ and (d) $\left(\sigma_{\epsilon}, \sigma_{\delta}\right)$ with $\sigma_{\epsilon}=0.5 \%$ and $\sigma_{\delta}=\sigma / K, \sigma=5 \%$ per annum and $K=250$ (corresponding to 250 trading days per year). The comparison is conducted over the first 500 time steps (a trading period of about 2 years). In all three noise cases, Fig. 5.1 (panel A) compares the market price $P_{t}$, together with the fundamental price and the long-run MA, Fig. 5.1 (panel B) compares the difference of the market population fractions $m_{t}=n_{f, t}-n_{c, t}$, and Fig. 5.1 (panel C) compares the demand functions of the fundamentalists and the technical analysts.

Case (a) reduces to the corresponding deterministic case. In this case, the constant fundamental price $P^{*}=100$ is unstable and the market price $P_{t}$ displays periodic switching between bull and bear markets, as illustrated in Fig. 5.1 (A)-(a). From Fig.5.1(C)-(a), one can see that the fundamentalists and the technical analysts take opposite (long/short) positions in most of 

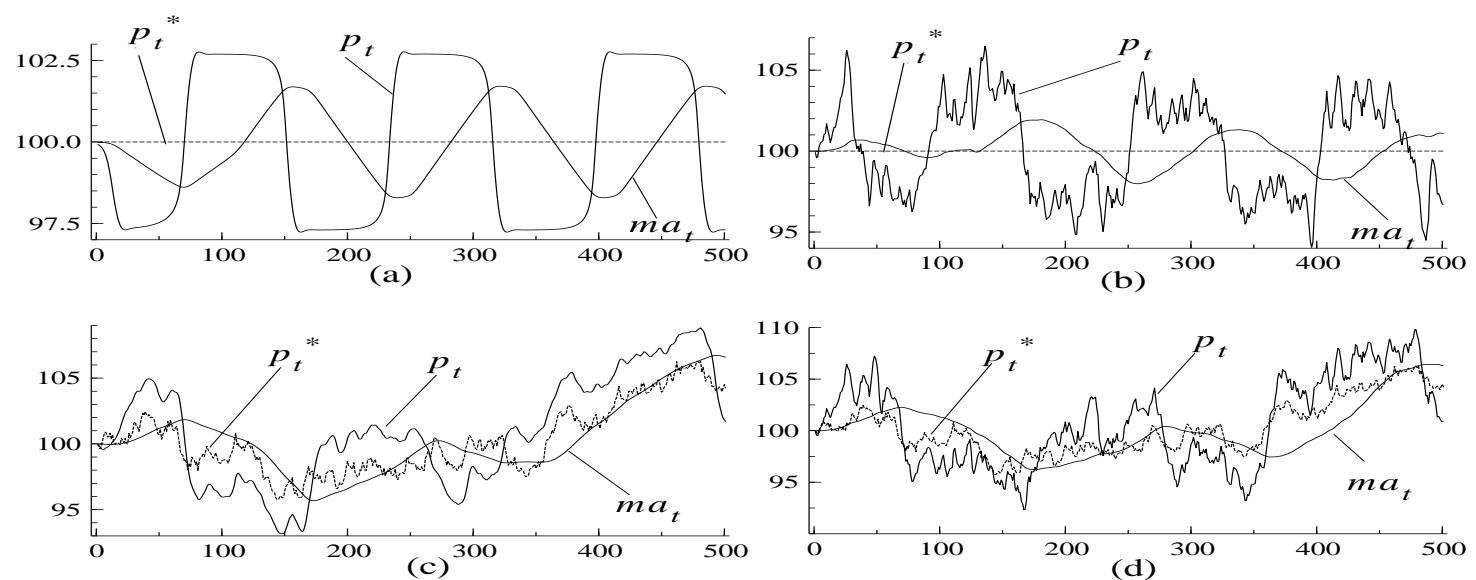

(A)
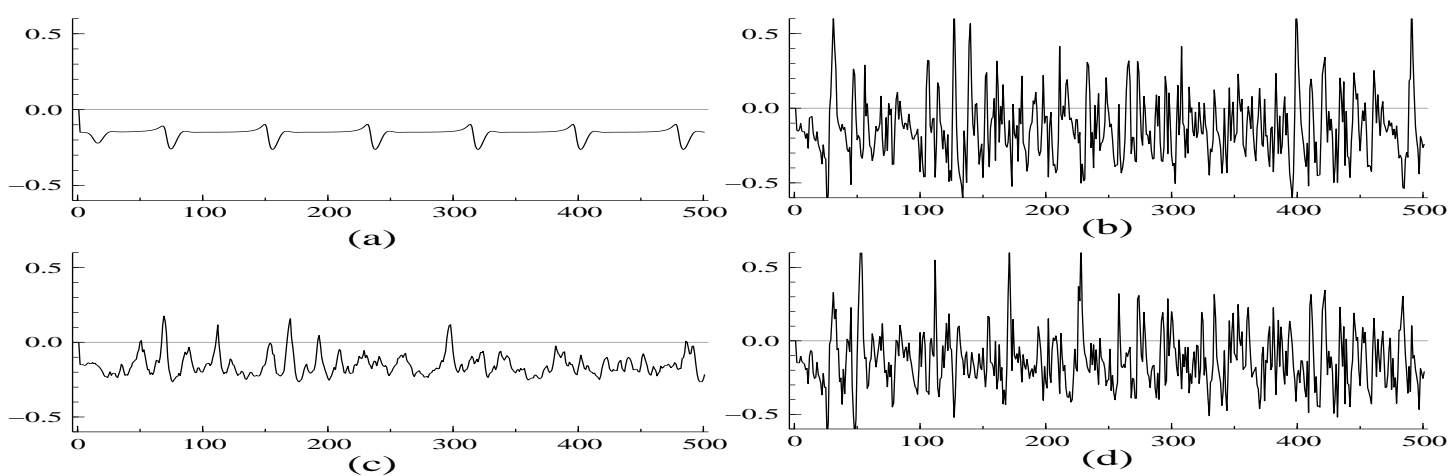

(B)
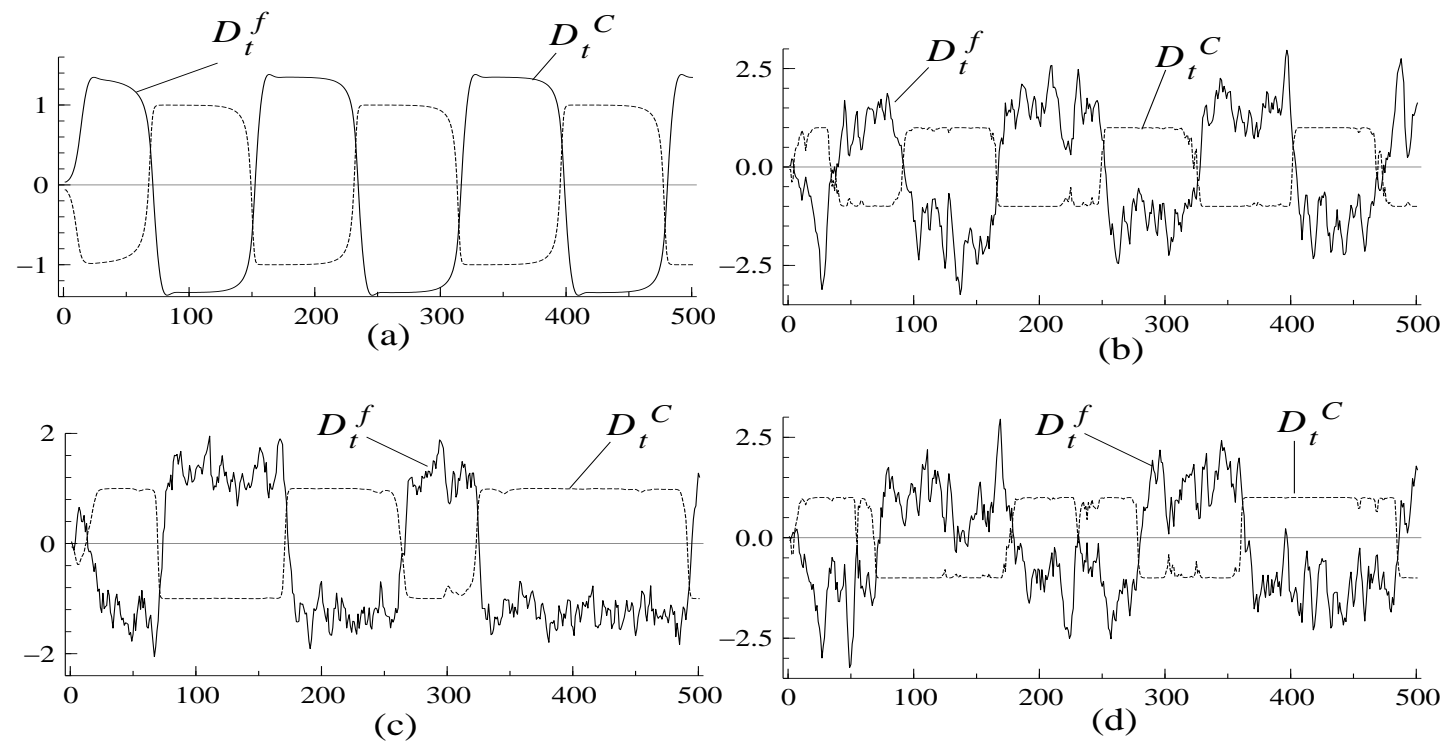

(C)

FigurE 5.1. Time series of the prices (A), population fraction differences (B), and demand functions (C) for fixed $L=100$ with $\left(\sigma_{\delta}, \sigma_{\epsilon}\right)=(0,0)$ in (a); $(0,0.5 \%)$ in (b); $(5 \%$ p.a., 0$)$ in (c) and (5\%p.a., $0.5 \%)$ in (d). Here $\alpha=0.5, \beta=$ $0.3, \mu=1, \eta=0.2, a=1, C=1$.

the time period. Because of limits on the position the technical analysts can take ${ }^{8}$ and the stabilizing role of the fundamentalists, such off-setting positions cause the price to stay bounded.

${ }^{8}$ This may be due to their short selling constraint when they hold a short position and consumption needs when they hold a long position. 
However the market switches when both of them have the same position and such a transition happens very quickly. In addition, the market is dominated by the technical analysts most of the time, as indicated by the fact that the trend of the market price in Fig. 5.1 (A)-(a) follows closely the demand pattern of the technical analysts in Fig. 5.1 (C)-(a) and that traders tend to switch from the steady state level to technical analysis as indicated by Fig. 5.1 (B)-(a).

Case (b) examines the effect of the noisy demand on the price dynamics. Because of this noisy demand, the market price becomes more volatile. However, the market price (in Fig.5.1 (A)-(b)) and the demand functions (in Fig. 5.1 (C)-(b)) are still dominated by the underlying price dynamics of the deterministic case (a), although the switching between two types of trading strategies is intensified (see Fig. 5.1 (B)-(b)), spreading between $m=-60 \%$ and $m=60 \%$.

Case (c) examines the effect of the noisy fundamental price on the price dynamics. One can see from Fig. 5.1 (A)-(c) that the market price $P_{t}$ closely follows the fundamental price $P_{t}^{*}$, though the variation of the market price increases (because of the strong extrapolation of the technical analysts). Fig. 5.1 (B)-(c) shows that traders tend to switch to fundamentalist analysis from time to time. However, a comparison of the market price trend in Fig. 5.1 (A)-(c) and the demand function pattern in Fig. 5.1 (C)-(c) shows that the market price is above (below) the fundamental price when the technical analysts take long (short) position. This means the market price is still dominated by the technical analysts although it follows closely the fundamental price.

Case (d) examines the combined effect of the two noise processes on the price dynamics. Apart from the fact that the market price becomes more volatile (because of the noisy demand), it shares similar features as in the cases (b) and (c). That is, the market price follows the fundamental price and the market is dominated by technical analysts.

Based on the analysis above, we observe some interesting phenomena. (i) Adding noisy demand can increase price volatility, but it has less impact on the price pattern and the market conditions of the underlying price dynamics. (ii) When the fundamental price follows a stochastic process, the market price closely follows the fundamental price. (iii) The market is mainly dominated by technical analysts (when they extrapolate strongly). They may be the winners over short time periods (indicated by the traders switching to technical analysis), however over the whole time period they may be the losers in the sense that most of the time they buy when the market prices are high and sell when the market prices are low. (iv) The switching between 
bull and bear markets happens when both types of traders take the same position, a very intuitive result. Such transitions can be intensified with the help of the noise traders, leading to temporary market bubbles and sudden crashes.

\section{Conclusions}

Within the framework of the Brock and Hommes (1998) asset pricing model with heterogeneous beliefs, price fluctuations are driven by evolutionary switching between different expectation schemes. Various rational routes to randomness, i.e. bifurcation routes to complicated dynamics, are observed when the intensity of choice to switch prediction strategies is high. In their framework however, the technical trading rules are very simple and for analytical tractability only a few lags are involved. Motivated by the popularity of MAs strategies in real markets and empirical studies, this paper sets out to analyze the impact of long run MA rules on the market dynamics and potentially rational routes to randomness. In our model of fundamentalists and technical analysts, who trade on the signals generated by the crossing of the latest price over the long run MA, we are able to obtain some important qualitative insights into the impact of MA rules. Intuitively one might expect that a long run MA smoothes the price dynamics and hence an increase of the lag length of the MA might be expected to stabilize the market. Surprisingly, our results show that, within a market maker scenario, this intuition is only true when both the reaction coefficient $\alpha$ of the fundamentalists and the extrapolation rate $a$ of the trend followers are balanced in a certain way. In general, as the lag length $L$ increases, the MA becomes smoother and more sluggish. When the impact between fundamentalists and chartists is not balanced, especially when the relative impact of chartists at the steady state is larger than that of fundamentalists, a mall change in the price leads to a relatively large increase of chartists demand and consequently, the lag length of the MA rule can destabilize the market price. To the best of our knowledge, this is a new result concerning market dynamics in the presence of MA rules. Another contribution of this paper is that for realistic MA rules with a large lag length $L$, similar rational routes to randomness occur when the intensity of choice to switch strategies increases. Finally, time series analysis of a stochastic version of our model shows the potential to explain various market phenomena such as price volatility, bull and bear markets, temporary bubbles and sudden crashes. In subsequent research it will be useful to study a more realistic model of the market with a large number of different trading rules, in particular with agents 
using different MA strategies of various length, or other types of technical trading rules used in financial practice, such as genetic algorithms and neural networks.

APPENDIX-PROOF OF PROPOSITION 3.1

A1. Existence and Uniqueness of the Steady-State-Proof of Part (i). The deterministic system (2.7)-(2.9) can be written as follows:

$$
P_{t+1}=F\left(X_{t}\right), \quad U_{t+1}=H\left(X_{t}\right), \quad m_{t+1}=G\left(X_{t}\right)
$$

where $X_{t}=\left(P_{t}, P_{t-1}, \cdots, P_{t-(L-1)}, U_{t}, m_{t}\right)$ and

$$
\begin{aligned}
& F\left(X_{t}\right)=P_{t}+\frac{\beta}{2}\left[-\left(1-m_{t}\right) \alpha\left(P_{t}-P^{*}\right)+\left(1-m_{t}\right) h\left(\psi_{t}^{L}\right)\right] \\
& H\left(X_{t}\right)=\left[-\alpha\left(P_{t}-P^{*}\right]-h\left(\psi_{t}^{L}\right)\right]\left[F\left(X_{t}\right)-P_{t}\right]+\eta U_{t} \\
& G\left(X_{t}\right)=\tanh \left[\beta\left(H\left(X_{t}\right)-C\right) / 2\right] .
\end{aligned}
$$

One can easily see that, for $\eta \in[0,1),\left(P_{t}, U_{t}, m_{t}\right)=\left(P^{*}, 0, m^{*}\right)$ is the unique steady state of the system (A.1), where $P^{*}$ corresponds to the constant fundamental price and $m^{*}=\tanh (-\beta C / 2)$.

A2. Characteristic Equation of the Steady-State. The characteristic equation of the system (A.1) at the steady state is given by $\Gamma(\lambda):=$ $\lambda(\lambda-\eta) \Gamma_{L}(\lambda)=0$ where

$$
\Gamma_{L}(\lambda):=\lambda^{L}-(1-\bar{\alpha}) \lambda^{L-1}-\bar{a}\left(1-\frac{1}{L}\right) \lambda^{L-1}+\frac{\bar{a}}{L}\left(\lambda^{L-2}+\cdots+\lambda+1\right)=0 .
$$

In fact, evaluated at the unique steady state, one can see that

$$
\begin{aligned}
\frac{\partial F}{\partial P_{t}} & =1+\frac{\mu}{2}\left[-\left(1+m^{*}\right) \alpha+\left(1-m^{*}\right) a\left(1-\frac{1}{L}\right)\right], \\
\frac{\partial F}{\partial P_{t-1}} & =\frac{\partial F}{\partial P_{t-2}}=\cdots=\frac{\partial F}{\partial P_{t-(L-1)}}=\frac{\mu}{2}\left(1-m^{*}\right) a\left(-\frac{1}{L}\right), \\
\frac{\partial F}{\partial U_{t}} & =\frac{\partial F}{\partial m_{t}}=0, \frac{\partial H}{\partial P_{t}}=\frac{\partial H}{\partial P_{t-1}}=\cdots=\frac{\partial H}{\partial P_{t-(L-1)}}=0, \\
\frac{\partial H}{\partial U_{t}} & =\eta, \frac{\partial H}{\partial m_{t}}=0, \frac{\partial G}{\partial P_{t}}=\frac{\partial G}{\partial P_{t-1}}=\cdots=\frac{\partial G}{\partial P_{t-(L-1)}}=0, \frac{\partial G}{\partial U_{t}}=\eta \beta / 2, \frac{\partial G}{\partial m_{t}}=0 .
\end{aligned}
$$

Based on these calculations, the result follows.

A3. Proof of Parts (ii) and (iii). The proofs of (ii) and (iii) follow from the following Lemma.

Lemma.(i) If $\bar{\alpha}=1+\bar{a}$, then the eigenvalues $\lambda_{i}$ of $\Gamma_{L}$ satisfy $\left|\lambda_{i}\right|<1$ if and only if $0<\bar{a}<L$. In addition, for $\bar{a}=L$, the $\lambda_{i}$ satisfy $\lambda_{i} \neq 1$ and $\left(1-\lambda_{i}^{L}\right) /\left(1-\lambda_{i}\right)=0$. (ii) A necessary condition for $\left|\lambda_{i}\right|<1$ for all $i$ is $0<\bar{a}<L$ and $0<\bar{\alpha}<2+\bar{a}$ for even $L$ and $0<\bar{\alpha}<2+\frac{L-1}{L} \bar{a}$ for odd $L$.

Proof. For $\bar{\alpha}=1+\bar{a}, \Gamma_{L}(\lambda) \equiv \lambda^{L}+\frac{\bar{a}}{L}\left(\lambda^{L-1}+\cdots+\lambda+1\right)=0$. It follows from Chiarella and He (2002) that $\left|\lambda_{i}\right|<1$ iff $-\frac{1}{L}<\frac{\bar{a}}{L}<1$, i.e., $\bar{a}<L$ (since $\bar{a}>0$ ). In general, following from Jury's test, necessary conditions for $\left|\lambda_{i}\right|<1$ for all $i$ are $\bar{a} / L<1, \Gamma_{L}(1)=\bar{\alpha}>0$ and $(-1)^{L} \Gamma_{L}(-1)=2-\bar{\alpha}+\bar{a}>0$ for even $L$ and $(-1)^{L} \Gamma_{L}(-1)=2-\bar{\alpha}+\frac{L+1}{L} \bar{a}>0$ for odd $L$.

A4. Proof of Part (iv). Let $f(\lambda)=\lambda^{L}$ and $g(\lambda)=-(1-\bar{\alpha}+\bar{a}) \lambda^{L-1}+\frac{\bar{a}}{L}\left[\lambda^{L-1}+\cdots+\lambda+1\right]$. Then, on $|\lambda|=1$, $|g(\lambda)|<|1-\bar{\alpha}+\bar{a}|+\bar{a}$ and $|f(\lambda)|=1$. If $2 \bar{a}<\bar{\alpha}<2$, then $|g(\lambda)|<|f(\lambda)|$ on $|\lambda|=1$. Following from Rouche's theorem, $f(\lambda)$ and $\Gamma_{L}(\lambda)=f(\lambda)+g(\lambda)$ have the same number of zeros inside $|\lambda|=1$. Therefore $\left|\lambda_{i}\right|<1$ for $i=1,2, \cdots, L$. 
A4. Proof of Part (v). To show that there exists at least one eigenvalue $\lambda_{o}$ such that $\left|\lambda_{o}\right|>1$ when $\bar{a}>\bar{\alpha}$ and $L$ is sufficiently large, we change variables by setting $\lambda=1+z / L$ in $\Gamma(\lambda)$ and introduce $f(z)=\lim _{L \rightarrow \infty} \Gamma(1+z / L)$. Note that for $|z|$ bounded, the limit is uniform. As $L \rightarrow \infty$, we have $\lambda^{L}=(1+z / L)^{L} \rightarrow e^{z}, \lambda^{L-1}=(1+z / L)^{L-1} \rightarrow e^{z}$ and $(1 / L) \sum_{i=0}^{L-1} \lambda^{i}=(1 / L)\left(\lambda^{L}-1\right) /(\lambda-1)=$ $\left[\left(1+z^{L}\right)^{L}-1\right] / z \rightarrow\left[e^{z}-1\right] / z$. Hence $f(z)=(\bar{\alpha}-\bar{a}) e^{z}+\bar{a}\left[e^{z}-1\right] / z$. Note that $f(0)=\bar{\alpha}>0$ and that $\lim _{z \rightarrow \infty}\left[\left(e^{z}-1\right) / z\right] / e^{z}=0$, therefore the first term in $f$ dominates the second. Since $\bar{\alpha}-\bar{a}<0$, we see that for large values of $z$ the value $f(z)$ will be negative. Consequently $f$ will have a positive zero, and $\Gamma$ will have a zero larger than one for $L$ sufficiently large.

A5. Proof of Part (vi)-(ix). For $L=1, \Gamma_{1}(\lambda) \equiv \lambda-(1-\bar{\alpha})=0$. Hence $|\lambda|<1$ iff $0<\bar{\alpha}<2$. Also $\lambda=+1$ for $\bar{\alpha}=0$ and $\lambda=-1$ for $\bar{\alpha}=2$.

For $L=2, \Gamma_{2}(\lambda)=\lambda^{2}+c_{1} \lambda+c_{2}=0$, where $c_{1}=-\left(1-\bar{\alpha}+\frac{1}{2} \bar{a}\right)$ and $c_{2}=\frac{\bar{a}}{2}$. Following Jury's test, $\left|\lambda_{i}\right|<1$ iff $\pi_{1}:=$ $1+c_{1}+c_{2}=\bar{\alpha}>0, \pi_{2}:=1-c_{1}+c_{2}=2-\bar{\alpha}+\bar{a}>0$ and $\pi_{3}:=1-c_{2}=1-\frac{\bar{a}}{2}>0$. Hence $P^{*}$ is LAS if $(\bar{\alpha}, \bar{a}) \in D_{12}(\bar{\alpha}, \bar{a})$. Also, $\lambda_{1}=1$ and $\left|\lambda_{2}\right|<1$ when $\pi_{1}=0, \lambda_{1}=-1,\left|\lambda_{2}\right|<1$ when $\pi_{2}=0$ and $\lambda_{1,2} \in C,\left|\lambda_{1,2}\right|=1$ when $\pi_{3}=0$.

For $L=3, \Gamma_{3}(\lambda):=\lambda^{3}-\left[1-\bar{\alpha}+\bar{a}\left(1-\frac{1}{3}\right)\right] \lambda^{2}+\frac{\bar{a}}{3}(\lambda+1)=0$. Set $c_{1}=-\left[1-\bar{\alpha}+\frac{2}{3} \bar{a}\right], c_{2}=c_{3}=\frac{\bar{a}}{3}$. Then $\left|\lambda_{i}\right|<1$ iff $\pi_{1}:=1+c_{1}+c_{2}+c_{3}=\bar{\alpha}>0, \pi_{2}:=1-c_{1}+c_{2}-c_{3}=2-\bar{\alpha}+\frac{2}{3} \bar{a}>0$ and $\pi_{3}:=1-c_{2}+c_{1} c_{3}-c_{3}^{2}=1-\frac{\bar{a}}{3}[2-\bar{\alpha}+\bar{a}]>0$. Hence $P^{*}$ is LAS if $(\bar{\alpha}, \bar{a}) \in D_{13}(\bar{\alpha}, \bar{a})$. Furthermore, $\pi_{1}=0, \pi_{2}=0$ and $\pi_{3}=0$ give the saddle-node, flip and Hopf bifurcation boundaries, respectively.

For $L=4, \Gamma_{4}(\lambda) \equiv \lambda^{4}-\left[1-\bar{\alpha}+\frac{3}{4} \bar{a}\right] \lambda^{3}+\frac{\bar{a}}{4}\left(\lambda^{2}+\lambda+1\right)=0$. Set $p=-\left[1-\bar{\alpha}+\frac{3}{4} \bar{a}\right], q=\frac{\bar{a}}{4}$. Then, using Jury's test, $\left|\lambda_{i}\right|<1$ iff $\Gamma_{4}(1)=\bar{\alpha}>0, \Gamma_{4}(-1)=2-\bar{\alpha}+\bar{a}>0, \bar{a}<4$ and both the determinants of the matrices

$$
A=\left(\begin{array}{ccc}
1 & 0 & q \\
p-1 & 1+q & 0 \\
2 q-p & p-1 & 1+p-q
\end{array}\right), \quad B=\left(\begin{array}{ccc}
1 & 0 & -q \\
p & 1-q & -q \\
0 & p-q & 1-p
\end{array}\right)
$$

are positive. It can be verified that $|A|>0,|B|>0$ iff $(1+q)^{2}[1+p-2 q]+q(p-1)^{2}>0$ and $p<1$, respectively, which leads to the result.

\section{REFERENCES}

Allen, H. and Taylor, M. (1990), 'Charts, noise and fundamentals in the London foreign exvhange market', Economic Journal 100, 49-59. Conference.

Arnold, L. (1998), Random Dynamical Systems, Springer-Verlag, Berlin.

Beja, A. and Goldman, M. (1980), 'On the dynamic behavior of prices in disequilibrium', Journal of Finance 35, 235-247.

Blume, L., Easley, D. and O'Hara, M. (1994), 'Market statistics and technical analysis: the role of volume', Journal of Finance 49, 155-181.

Boswijk, H., Griffioen, G. and Hommes, C. (2000), Succes and failure of technical trading strategies in the cocoa futures markets, CeNDEF Working paper 00-06, University of Amsterdam.

Brock, W. and Hommes, C. (1997), 'A rational route to randomness', Econometrica 65, 1059-1095.

Brock, W. and Hommes, C. (1998), 'Heterogeneous beliefs and routes to chaos in a simple asset pricing model', Journal of Economic Dynamics and Control 22, 1235-1274.

Brock, W., Lakonishok, J. and LeBaron, B. (1992), 'Simple technical trading volatility and the stochastic properties of stock returns', Journal of Finance 47, 1731-1764.

Brock, W. and LeBaron, B. (1996), 'A structural model for stock return volatility and trading volume', Review of Economics and Statistics 78, 94-110.

Brown, D. and Jennings, R. (1989), 'On technical analysis', The Review of Financial Studies 2, 527-551.

Chiarella, C. (1992), 'The dynamics of speculative behaviour', Annals of Operations Research 37, 101-123.

Chiarella, C. and He, X. (2000), The Dynamics of the Cobweb when Producers are Risk Averse Learners, PhysicaVerlag, pp. 86-100. in Optimization, Dynamics, and Economic Analysis, E.J. Dockner, R.F. Hartl, M. Luptacik and G. Sorger (Eds). 
Chiarella, C. and He, X. (2002), 'Heterogeneous beliefs, risk and learning in a simple asset pricing model', Computational Economics 19, 95-132.

Chiarella, C. and He, X. (2003a), 'Dynamics of beliefs and learning under $\mathbf{a}_{l}$-processes - the heterogeneous case', Journal of Economic Dynamics and Control 27, 503-531.

Chiarella, C. and He, X. (2003b), 'Heterogeneous beliefs, risk and learning in a simple asset pricing model with a market maker', Macroeconomic Dynamics 7, 503-536.

Day, R. and Huang, W. (1990), 'Bulls, bears and market sheep', Journal of Economic Behavior and Organization 14, 299-329.

Fama, E. (1970), 'Efficient capital markets: a review of theory and empirical work', Journal of Finance 25, 383423.

Fernandez-Rodriguez, F., Gonzalez-Martel, C. and Sosvilla-Rivero, S. (2000), 'On the profitability of technical trading rules based on artificial neural networks: Evidence from the Madrid stock market', Economics Letters 69, 89-94.

Frankel, F. and Froot, K. (1986), 'Understanding the US dollars in the eighties: the expectations of chartists and fundamentalists', Economic Record, Supplementary Issue 62, 24-38.

Frankel, F. and Froot, K. (1990), Private Behaviour and Government Policy in Interdependent Economies, Vol. A.S. Courakis and M.P. Taylor (eds), Oxford University Press, chapter "Chartists, fundamentalists and the demand for dollars".

Gencay, R. (1998), 'Optimization of technical trading strategies and the profitability in security markets', Economics Letters 59, 249-254.

Goldbaum, D. (2003), 'Profitable technical trading rules as a source of price instability', Quantitative Finance 3, 220-229.

Griffioen, G. (2003), Technical Analysis in Financial Markets, Ph-D thesis, TI research series 305, University of Amsterdam.

Hale, J. and Kocak, H. (1991), Dynamics and bifurcations, Vol. 3 of Texts in Applied Mathematics, Springer-Verlag, New York.

Hommes, C. (2005), "Heterogeneous Agent Models in Economics and Finance", chapter in: Judd, K.L. and Tesfatsion, L. (eds.) Handbook of Computational Economics Vol. 2: Agent-based Computational Economics, North-Holland, to appear.

Kuznetsov, Y. (2004), Elements of applied bifurcation theory, Vol. 112 of Applied mathematical sciences, 3rd edn, SV, New York.

LeBaron, B. (2005), “Agent-based Computational Finance", chapter in: Judd, K.L. and Tesfatsion, L. (eds.) Handbook of Computational Economics Vol. 2: Agent-based Computational Economics, North-Holland, to appear.

Lo, A., Mamaysky, H. and Wang, J. (2000), 'Foundations of technical analysis: computational algorithms, statistical inference, and empirical implementation', Journal of Finance 55, 1705-1770.

Manski, C. and McFadden, D. (1981), Structural Analysis of Discrete Data with Econometric Applications, MIT Press.

Neely, C. (1997), 'Technical analysis in the foreign exchange market: A layman's guide', Federal Reserve Bank of St. Louis Review (Sept./Oct.), 23-38.

Neely, C., Weller, P. and Dittmar, R. (1997), 'Is technical analysis in the foreign exchange market profitable? a genetic programming approach', Journal of Quantitative and Financial Analysis 32, 405-426.

Peitgen, H.-O., Jurgens, H. and Saupe, D. (1992), Chaos and Fractals—New Frontiers of Science, Springer-Verlag, New York.

Pesaran, M. and Timmermann, A. (1994), 'Forecasting stock returns, an examination of stock market trading in the presence of transaction costs', Journal of Forecasting 13, 335-367.

Pesaran, M. and Timmermann, A. (1995), 'Predictability of stock returns: Robustness and economic significance', Journal of Finance 50, 1201-1228.

Pring, M. (1991), Technical Analysis Explained, 3rd edn, McGraw-Hill, New York.

Sonis, M. (2000), 'Critical bifurcation surfaces of 3d discrete dynamics', Discrete Dynamics in Nature and Society 4, 333-343.

Taylor, M. and Allen, H. (1992), 'The use of technical analysis in the foreign exchange market', Journal of International Money and Finance 11, 304-314. 